\title{
Revision eines klassischen Falles von Diverticulum des Oesophagus.
}

Von Dr. C. D. Spivak in Denver, Colo., U. S. A.

Unter Divertikel des Oesophagus verstehen wir bekanntlich eine Erweiterung der Wand der Speiseröhre mit Bildung eines Blindsackes. v. Zenker und v. Ziemssen waren die ersten, die diese Divertikel klassifizierten. Ein pathologischer Zustand entzündlicher Natur in einem der anliegenden Organe oder Gewebe außerhalb des Oesophagus übt gelegentlich einen Zug auf die Wand des letzteren aus und bringt somit eine Ausbuchtung zusstande. Dies führt zur Hervorbringung des sogenannten Traktions-Divertikels. Ein pathologischer Zustand innerhalb des Lumens des Oesophagus selbst kann eine Erweiterung in einem bestimmten Teile der Oesophagealwand, unmittelbar oberhalb 
der Läsion, zur Folge haben, und dies nennt man dann PulsionsDivertikel.

Diese beiden Arten von Divertikeln unterscheiden sich voneinander nicht nur in bezug auf ihre Pathogenese, sondern auch hinsichtlich ihrer Frequenz, Morphologie, Lokalisation und Symptomatologie.

Ein Zug- oder Traktions-Divertikel hat seinen Sitz in de vorderen Wand der Speiseröhre, an der Bifurkationsstelle der Trachea. Es ist trichterförmig und 2-8 $\mathrm{cm}$ tief. Da es gewöhnlich ohne jedwede Symptome verläuft, so wird es bei Lebzeiten nicht erkannt.

Das Pulsions - Diver tikel ist im Zervikalabschnitt des Oesophagus lokalisiert. Sein Eingang liegt unmittelbar an der Uebergangsstelle zwischen Pharynx und Oesophagus; es wird daher als Pharyngo-Oesophageal-Divertikel bezeichnet. Es stellt eine birnförmige Ausbuchtung von $13 \mathrm{~cm}$ und mehr Tiefe dar und gibt Veranlassung zll einer Reihe von bestimmten Symptomen, unter welchen Dysphagie das hervor ragendste ist. In den letzten Stadien der Krankheit, wenn der Blindsack mit Speisestoffen gefüllt ist, kann eine umschriebene oder diffuse Schwellung auf der Rückseite des Oesophagus in dieser Gegend beobachtet werden. Diese verschwindet nach Entleerung der Speisestoffe durch Frbrechen oder durch künstliche Entleerung des Blindsacks. Fs ist eine Krankheit des vorgerïickten Alters.

Dies sind der Hauptsache nach die diagnostischen Punkte, welche die genannten zwei Divertikelarten, wie sie von den beiden erwähnten Gelehrten beschrieben worden sind, voneinander unterscheiden. Die Zenker-Ziemssensche Klassifikation wurde von allen Pathologen und Klinikern einwandfrei angenommen und stand als unerschüttert da, bis vor 15 Jahren von Dr. Z. B ychowsky in Warschau nachfolgender Fall in Virchows Archiv, Band 141 veröffentlicht wurde.

„P. W., 21 Jahre alt, Schneidergehilfe. Seit neun Jahren Erbrechen, sowohl nach eingenommener Nahrung als manchmal auch während der Mahlzeit. Das Erbrochene besteht aus den verschluckten Speisen. Unbedeutendes Druckgefühl sofort nach dem Essen, und zwar in der Gegend des Sternum. Weder Aufstoßen nocl Sodbrennen. Appetit immer gut, Darmfunktionen regelmäßig. Patient fühlt sich nicht sclıwach, arbeitet zehn bis zwölf Stunden täghich.

Status. Ernährungszustand befriedigend. Wiegt $54 \frac{1}{2} \mathrm{~kg}$. Haut. decken schmutzig gelblich. Keine Zähne. Laryngoskopische Untersuchung negativ. Halsumfang normal. Keine Narben. Herz und Lungen ohne Veränderung. Ueber dem Thorax keine Dämpfung. Abdomen nicht erweitert, nicht druckempfindlich. Kein Sukkussionsgeräusch. Untere Grenze des Magens oberhalb des Nabels. Urin eiweiß. und zuckerfrei. Spuren von Indikan. Kniereflexe prompt, jedoch nicht gesteigert. Hautreflexe normal. Keine Zeichen von angeborener oder erworbener Syphilis.

Magenuntersuchung (nüchtern): Ein gewöhnliches Bougie geht ohne Schwierigkeiten durch. Durch dasselbe werden etwa $100 \mathrm{ccm}$ einer schmutziggelben, dicklichen, fast geruchlosen Flüssigkeit von normaler Reaktion herausbefördert. Mikroskopisch zahlreiche Bruchstücke von Nahrungsstoffen und Fetzen von geschichtetem, schwainmigem Epithel Keine Sarzine. Während der folgenden Untersuchungen wurde noch mehrmals Milchsäure, jedoch keine Salzsäure nachgewiesen.

Ein gewöhnliches weiches Magenrohr gelangt in einer Entfernung von $40 \mathrm{~cm}$ von den Schneidezähnen auf ein unïberwindliches Hindernis Wird der Versuch gemacht, das letztere mit Gewalt zu durchbrechen, so biegt sich das Rohr um seine eigene Achse, und das untere Rohrende kommt schließlicl durch den Mund zurück. Ein hartes Bougie geht nicht weiter als 36 bis $37 \mathrm{~cm}$. Durch ein gewöhnliches Rohr kann man allmählich 250 bis $300 \mathrm{ccm}$ Flüssigkeit in den Blindsack hineinpumpen, welche durch die üblichen Manipulationen beim Auswaschen des Magen. zurückbefördert werden kann. Werden jedoch mehr als $300 \mathrm{ccm}$ lineingegossen, so kommt die Flüssigkeit durch den Mund spontan zurück Patient kann zwischen 250 und $275 \mathrm{ccm}$ warıner Flüssigkeit langsam und ohne $\mathrm{zu}$ erbrechen verschlucken. - Die angewandte Flüssigkeit wurde mit Rotwein oder mit Tee gefärbt. Ein Teil derselben wurde be jedem Versuche zur Kontrolle zurückgelassen. Die durch das Magen rohr entfernte Flüssigkeit behielt stets ihre Farbe, war jedoch etwas mehr trübe. - Ein elektrisches Bougie blieb gewöhnlich an derselben Stelle stecken wie die anderen Instrumente. Durchleuchtungsversuche negativ. Die Diagnose eines Oesophagus-Divertikels war so mit bewiesen. Es schien etwa 20 bis $22 \mathrm{~cm}$ von den Schneidezähnen seinen Anfang zu nehmen, da der Inhalt des vollgefüllten Divertikels zurückkam, sobald das Magenrohr eine Entfernung von 22 bis $25 \mathrm{~cm}$ von den Schneidezähnen erreichte. Das untere Ende des Divertikels mußte 36 bis $37 \mathrm{~cm}$ von den Schneidezähnen entfernt sein, somit seine Tiefe 14 bis $17 \mathrm{~cm}$ und die Kapazität von 250 bis $300 \mathrm{ccm}$ betragen. - Aufblasen des Divertikels brachte keine Veränderung in der äußeren Gestalt des Halses hervor. Einmal gelang es dem Verfasser, in den Magen hineinzukommen und eine Flüssigkeit zu entfernen, welche gegen sämtliche bei der Untersuchung des Mageninhalts angewandte Proben sauer reagierte. Dieselbe Flüssigkeit, einige Minuten zuvor aus dem Divertikel herausbefördert, reagierte auf keine dieser Proben. Symptome einer Stenose des Oesophagus lagen nicht vor." -
Obenangefülurte Angaben sind die wichtigsten Punkte in dem Berichte des Verfassers. Der Fall wurde von ihm so sorgfältig studiert, so unständlich beobachtet, die Data waren so klar dargelegt und die Schlußfolgerungen so logisch gezogen, daß sie eine sofortige und allgemeine Anerkennung von seiten der Pathologen, Kliniker und Chirurgen fanden. Eine dritte Form von Oesophagus-Divertikel wurde anerkannt, nämlich ein tief.sitzendes oder strikt ösophageales Divertikel, zum Unterschied von dem Zenker - Ziemssenschen pharyngo-ösophagealen. Außerdem hatte der Verfasser mit seinem Falle bewiesen, daß ein Pulsions-Divertikel auch bei sehr jungen Individuen vorkommen kann. Sämtliche Lehrbücher der Chirurgie (v. Bergmann, Keen etc.) berufen sich auf diesen Fall, und in den Literaturangaben, welchen Aufsätze über diesen Gegenstand beigefügt werden, nimmt der Name des Verfassers eine Ehrenstelle ein.

14 Jahre nach Veröffentlichung dieses Falles brachte das Schicksal den Helden der Geschichte wegen eines Lungenleidens nach Colorado. Er tritt in das Sanatorium der ,Jüdischen Gesellschaft zur Unterstützung von armen Schwindsüchtigen" (Jewish Consumptives' Relief Society) in Edgewater bei Denver ein und gelangt hier wegen seines Oesophagus leidens in die Hände des Verfassers vorliegender Zeilen. Letzterer versichert wie sein Vorgänger in Virchows Archiv, daß er den Patienten äußerst sorgfältig und lege artis untersucht und beobachtet hat, und gestattet sich mun, dieselbe Geschichte größtenteils umgeschrieben und ınit einem vollständig neuen Hintergrunde den Lesern dieser Zeitschrift vorzulegen. Mein Vorgänger wird seinen Fall kaum erkennen.

Im zwölften Lebensjahr nach Erkältung plötzliche Unfähigkeit zu schlucken. Jeder Bissen kam sofort zurück. In den nächsten sechs Jahren ernährte der Patient sich liauptsächtlich von Weißbrot, Tee und großen Mengen Zucker. Im Alter von 18 Jahren ereignete es sich, daß ein Bissen Fleisch, ,in seinem Halse" stecken blieb. Erleichterung erfolgte erst am dritten Tage, nachdem or den Fleischbissen erbrochen hatte. Dr. Reichmann in Warschau behandelte ilın ein ganzes Jahr, doch ohne Erfolg. Er kam darauf in die Behandlung von Dr. B ychowsky, dessen Beobachtungen wir oben wiedergegeben haben. Dann kam er nach Berlin; zu der von Prof. Koenig vorgeschlagenen Operation wollte er sich nicht entschließen und begab sich von dort nach Paris. Er fand dort durch Erfahrung lieraus, daß Wein vermischt mit Wasser die Bissen schlüpfriger und durchgängiger machte. Als er im Jahre 1900 nach den Vereinigten Staaten kam, vertauschte er das Weintrinken mit Bier, schließlich mit gewöhnlichem Trinkwasser. Er trinkt mit jeder Mahlzeit zwei bis vier Glas Wasser schluckweise und bewässert sonit jeden Bissen, bevor er ihn herunterschluckt. Das Wasser ınu von einer bestimmten Temperatur sein: wenn zu kalt oder zu heiß, so hilft es nicht den Bissen nach unten befördern. Eine größere Quantität Wasser kann er nicht zu sich nehmen. Trinkt er Milch, Kaffee und dergleichen, so ist er ge nötigt, Wasser nachfolgen zu lassen, denn sonst bleibt der Bissen stecken. Er kann die Nahrung mehrere Tage nacheinander behalten, wenn er nur vorsichtig genug ist, sie gehörig mit Wasser zu durchtränken. Gelegentlich befördert er eine Stunde oder länger nach der Mahlzeit etwas Flüssigkeit heraus, auch wenn er wälırend und nach dem Essen keine Beschwerden empfand. In den letzten zehn Jahren erbricht er oft des Morgens eine glasige, saure Flüssigkeit. Unter allen Fleischarten macht ihm Rindfleisch am meisten Beschwerden beim Schlucken, rohes Fleisch ain wenigsten. Früchte kann er nur nach Entfernung der Rinde verschlucken. Besonders vorsichtig muß er mit Kartoffeln sein. Er verwendet ungefähr zweimal soviel Zeit für seine Mahlzeiten als seine Tischgenossen.

Seitdem der Patient in meine Obhut gelangt ist, liabe ich an ihm folgende Beobaclıtungen machen können.

Bei Einführung eines Magenrohres in den Oesophagus stößt man $40 \mathrm{~cm}$ hinter den Schneidezähnen auf einen Widerstand. Es werden von 60 bis $90 \mathrm{ccm}$ alkalische, geruchlose Flüssigkeit herausbefördert. Nach einer Reihe von unfruclıtbaren Bemühungen konstruierte ich ein eigenes Bongie aus einem Seildraht, an dessen distalem Ende ein äußerst kleiner olivenförmiger Ansatz angebraclit war. Dieses ging unter einem leichten Druck glatt bis zu einer Tiefe von $50 \mathrm{~cm}$ durch. In den darauffolgenden Tagen wurde das Bougie mehrere Male eingeführt, und zwar mit demselben Erfolg mit Bezug auf die Ueberwindung der Obstruktionsstelle. Es war nun klar, daß dieser Erfolg von keinem Zufall abhing. Ich wußte jetzt mit Sicherheit, $\mathrm{da} B$ ich nicht den Boden eines Divertikels sondierte, sondern daß ich -einfach einen Widerstand im Oesophagus passierte.

Der Versuch jedoch, ein Magenrohr durchpassieren zu lassen, wollte mir auch jetzt nicht gelingen. Ich führte nun das Drahtbougie in den Magen ein, stülpte darüber das Magenrohr und fuhr fort, dieses vorwärts zu schieben, worauf das Rohr in den Magen mit derselben Leichtigkeit hineingelangte wie das Bougie selbst. Derselbe Erfolg ergab sich bei jedesmaliger Wiederholung des Versuchs. Nun entfernte ich den Draht, und es gelang mir, aus dem Magen den Inhalt einer Probemahlzeit herauszubekommen, bei dessen Untersuchung die Gegenwart von Salzsäure festgestellt werden konnte. Der Magen wurde dann aus. gewaschen. 
Dr. Childs in Denver war so freundlich, ein Röntgenbild aufzunelhmen, nachdem der Patient Bismutnitrat in Kapseln und in Emulsion bekommen, sowie nachdem er eio goldenes Kettchen verschluckt hatte. Sämtliche Bilder zeigten, daß das Bismut längs dem Verlaufe des Oesophagus verbreitet war.

Wir haben also vor uns einen Fall von Dysphagie, welche seinerzeit plötzlich und heftig einsetzte und sich im Lanfe der Zeit von selbst und ohne irgendwelche besondere Behandlung besserte, - eine Dysphagie, welche nacli 23 jährigem Bestand weder das Körpergewicht des Patienten reduziert, noch seine Gesundheit in allgemeinen untergraben hat. Dies spricht zweifellos gegen das Bestehen eines Divertikels!

Zwei andere Zustände konnten hier vorliegen, nämlich eine Stenose auf organischer oder funktioneller Grundlage, oder eine diffuse Dilatation des Oesophagus. Daß eine Sten ose des Oesophagus vorliegt, kann kaum bezweifelt werden. Die Stenose ist hier tastbar. Es ist aucl klar, daß ein organischer Ursprung dieser Stenose ausgeschlossen werden kann, da diese so viele Jahre bestand, ohne sich verschlimmert zu haben. Im Gegenteil, sie zeigte zu verschiedenen Perioden spontane Besserung. $\mathrm{Zu}$ gunsten einer diffusen Dilatation des Oesophagus spricht nur eine einzige Tatsache, und zwar die Angabe von Dr. B y chowsky, daß früher einmal der Oesophagus imstande war, eine ziemlich große Menge Flüssigkeit zu behalten. Da ich selbst seinen vor 14 Jahren erhobenen Befund nicht. bestätigen konnte, so muß ich annehmen, daß die ausgedehnten Wände des Oesophagus in der Zwischenzeit in ihre normale Lage zurückgekehrt waren, eine Annahme, welche kaum stichhaltig ist. Sollten wir aber auch gewillt sein anzuerkennen, daß sogar jetzt noch eine leichte diffuse Dilatation des Oesophagus bestehe, so versichern uns sämtliche Autoren, die solche Fälle früher beschrieben haben, daß sie den Oesophagus stets durchgängig gefunden haben und daß eine Stenose in Verbindung mit einer diffusen Dilatation nie gefunden worden ist.

Es bleibt somit für die Diagnose nur die eine Annahme, nämlich daß hier eine funktionelle Stenose, oder mit anderen Worten, Kardio spasmus vorliegt. Auf diese Diagnose deuten hin: das plötzliche Einsetzen, das jugendliche Alter des Patienten zu der Zeit, als er zuerst von seinem Leiden befallen wurde, die Perioden des Verschwindens sämtlicher Symptome, besonders aber die Abwesenheit von Erbrechen, das eigentümliche Verhalten des Oesophagus gegenüber bestimmten Speisen. Huhn z. B. kann er leichter verschlucken als Rindfleisch, Kaffee muß er von hinten mit einem Schluck Wasser nachschieben, einige wenige Kartoffelstärkekörnchen bleiben stets im Schlunde stecken. Und endlich die große Empfindlichkeit der Schleimhaut gegen bestimmte Temperaturen, wobei selır kaltes oder sehr warmes Wasser das Vordringen der Speisen verhindert.

Es gibt eine Reile von ähnlichen Fällen in der Literatur, die zu einer Revision der Diagnose auffordern. Eben weil der' vorliegende Fall die Basis zu einer neuen Klassifikation der pathologischen Zustände im Oesophagus abgibt, außerdem als ,klassischer" Fall in den Lehrbüchern und in Monographien von Autoritäten zitiert wird und sich endlich nach 14 Jahren als eine Fiktion entpuppt hat, verdient er unzweifelhaft, veröffentlicht zu werden. Ich gebe mich der Hoffnung hin, daß Dr. Bychowsky sowie die sämtlichen Autoren neuer Auflagen von medizinischen und chirurgischen Lehrbüchern nicht verfehlen werden anzuerkennen, daß in dem hier ausführlich berichteten Falle bisher ein gewaltiger Irrtum vorgelegen hat. 